\title{
Differences in Alcohol Consumption and Preferences for Types of Alcoholic Beverages in OECD Countries
}

\author{
GAVUROVÁ, B. ${ }^{1}$, RIGELSKÝ, M. ${ }^{2}$, IVANKOVÁ, V³ .
}

1 | Tomas Bata University, Faculty of Management and Economics, Centre for Applied Economic Research, Zlín, Czech Republic

2 University of Prešov, Faculty of Management, Prešov, Slovak Republic

3 | University of Prešov, Faculty of Management, Department of Economics and Economy, Prešov, Slovak Republic
Citation | Gavurová, B., Rigelský, M., Ivanková, V. (2020). Differences in Alcohol Consumption and Preferences for Types of Alcoholic Beverages in OECD Countries. Adiktologie, 2011-2), 29-36; doi 10.35198/01-2020-001-0001.
BACKGROUND: Increased alcohol consumption is generally considered to be a very negative matter affecting the socio-economic status of countries. Different types of alcohol pose different risks.

Therefore, mapping the alcohol consumption and alcohol preferences of different countries is a very important step in the process of reducing geographical disparities. AIM: The primary objective of this study was to determine the differences in alcohol consumption and alcohol preferences in OECD countries. DESIGN AND MEASUREMENT (METHODS): Non-parametric analysis of difference (Kruskal-Wallis test) and cluster analysis (hierarchical clustering - Ward's method) were used to achieve the main objective. SAMPLE (PARTICIPANTS): The analytical process involved 36 OECD countries and the data that was analysed was divided into the following main categories of alcohol consumption: (i) beer, (ii) wine, (iii), spirits, (iv) other, and (v) all. Data was collected from 2010 to 2018.

RESULTS: The differences between the countries that were analysed were significant for all the selected variables (beer, wine, spirits, other, and all), but there was no significant difference between the years that were analysed. The findings show that the highest total alcohol consumption was in Estonia. The Czech Republic showed the highest beer consumption and France was the dominant country in wine consumption. The highest consumption of spirits was found in Estonia and other types of alcoholic beverages were preferred in Korea. The highest total alcohol consumption was found in Eastern Europe. CONCLUSIONS: This study highlighted the need for an effective intervention strategy to control alcohol consumption in developed countries. Drinking spirits and beer is the riskiest, and therefore education and prevention should focus on areas with a higher consumption of these types of alcoholic beverages.

Keywords | Alcohol Consumption - Alcoholic Beverages - Beer - Wine - Spirits - Regional Differences - OECD

Grant affiliation | This research was supported by the Internal Grant Agency of the Faculty of Management and Economics of Tomas Bata University in Zlín: RV0/2020: "Economic quantification of marketing processes that focus on the increase in value for a patient in the process of the creation of a system to measure and control efficiency in health facilities in the Czech Republic". 


\section{INTRODUCTION AND THEORETICAL BACKGROUND}

At present, alcohol consumption has become a habit that is also influenced by societal norms or cultural traditions, but in some cases this habit can become addictive and harmful. A specific feature of this issue is the fact that alcohol is commonly available and promoted and accepted in many communities, and at the same time its cost may not be high. On the other hand, alcohol can be perceived as a serious problem of the population as it significantly affects the health of people, which can manifest itself in diseases and disabilities, but also deaths (Rehm et al., 2010; Smyth et al., 2015). It is known from many studies that alcohol consumption is associated with a higher risk of mortality from specific diseases such as cirrhosis, cancer, and injuries (Klein et al., 2020; Roerecke et al., 2019; Thun et al., 1997). Scherubl (2019) also revealed that higher alcohol consumption leads to a higher risk of cancer, especially oropharyngeal and laryngeal cancer, oesophageal squamous cell cancer, hepatocellular carcinoma, breast cancer, and colorectal cancer. Similar findings were recorded by other authors such as Pelucchi et al. (2011), Bagnardi et al. (2013), and Scoccianti et al. (2013). At the same time, there is evidence that alcohol consumption is a risk factor not only for cancer, but also for cardiovascular and liver disease (McClure et al., 2013). On the evidence of these and many other findings, alcohol is a serious threat to public health and a risk factor for the global mortality burden (Rehm \& Shield, 2013). In another respect, alcohol consumption represents an economic burden, which is reflected in increased direct and indirect costs such as healthcare expenditure, lost productivity, and decreased gross domestic product (GDP) (Jyani et al., 2019; Laramee et al., 2013; Ranaweera et al., 2018; Thavorncharoensap et al., 2010). According to Verhaeghe et al. (2017), the most significant negative aspects of alcohol consumption include the costs associated with hospitalization, premature mortality, and disability-adjusted life years. Lyszczarz (2019) also examined alcohol consumption, alcohol-related mortality, and productivity losses in the European Union, and the findings of this study showed that there are differences between countries, with countries with higher alcohol consumption suffering higher total productivity losses as a result of alcohol-related mortality. It should be noted that in the European Union, the number of alcohol-related deaths in the working-age population was estimated at 137,122 in 2016, with Germany, Poland, and France showing the highest numbers of deaths resulting from alcohol consumption. On the other hand, the lowest numbers were found in Malta, Cyprus, and Luxembourg. At the same time, Lithuania, Estonia, and Latvia were identified as countries with the highest per capita costs of alcohol-related mortality. At the opposite end were countries such as Malta, Greece, and Italy. The OECD (2019) warns against high alcohol consumption, while in 2007-2017 Lithuania, Austria, France, and the Czech Republic were among the OECD countries with the highest alcohol consumption, and conversely, Turkey, Israel, and Mexico were among the countries with the lowest alcohol consumption.

Differences in alcohol consumption are evident not only at the level of countries or regions (Branstrom \& Andreasson, 2008; Bencevic-Striehl et al., 2009), but also in connection with the place where alcohol consumption takes place (Callinan et al., 2016) or the type of alcoholic beverage (Kossova et al., 2017). The consumption of individual types of alcoholic beverages poses different risks. In a Swiss study Dey et al. (2014) point to the fact that a preference for beer is riskier from a health point of view than a preference for wine, and at the same time a preference for beer can be expected to increase the incidence of smoking or the consumption of illegal substances. Kerr et al. (2000) also found that the consumption of spirits is more associated with mortality from cirrhosis in comparison with beer and wine. In a study conducted by Bentzen and Smith (2018), 21 OECD countries were divided into three groups according to their alcoholic beverage type preferences: beer-oriented countries (Austria, Belgium, Denmark, Ireland, the Netherlands, the United Kingdom, the United States, Australia, New Zealand), wine-oriented countries (France, Greece, Italy, Portugal, Spain, Switzerland), and spirits-oriented countries (Finland, Norway, Sweden, Japan). The authors further pointed out that since 1960, these OECD countries have converged in terms of alcohol consumption and the preferred alcohol type among the population. This fact was mainly due to the development of incomes (Bentzen \& Smith, 2018). On the basis of the findings revealed by Ogwang and Cho (2009), it can also be highlighted that in the case of the consumption of wine and spirits, income seems to be an important determinant. On the other hand, unemployment is a significant determinant of beer consumption. In any case, socio-economic status and level of education seem to be important aspects of the patterns of alcohol consumption (Devaux \& Sassi, 2015). On the other hand, Probst et al. (2020) revealed that alcohol consumption can explain socio-economic inequalities in alcohol-related mortality as well as all-cause mortality.

On the evidence of the above-mentioned findings, alcohol-related mortality represents a major social and economic burden and for this reason, Spach (2016) emphasized active steps of public policies in the area of alcohol consumption. Mehta and Myrskyla (2017) found that a healthy lifestyle that includes lower alcohol consumption has a positive effect on people's life expectancy. At this point, public policy can be very powerful; there are many effective tools that can reduce alcohol consumption. Anderson et al. (2009) found that high prices, a ban on alcohol advertising, and taxes can lead to a reduction in alcohol-related harm. In this respect, it is necessary to identify alcohol consumption in countries in order to determine the differences and to support preventive measures in developed countries. This study provides an overview of alcohol consumption and alcoholic beverage preferences in all OECD countries from 2010 to 2018. The core of the main idea of this study is to emphasize the need for an effective intervention strategy to control alcohol consumption in developed countries. 


\section{METHODOLOGY}

As mentioned in the previous section, alcohol consumption has demonstrable effects on several areas of health. The preferences for certain types of alcoholic beverages do not have the same effects on the health, or rather on the morbidity, of the population. At the same time, there is a different incidence of combining the preferred type of alcohol with other substances (e.g. smoking). Therefore, it is at least appropriate to point out the differences in alcohol consumption in geographical terms. The primary objective of the present study is to determine the differences in alcohol consumption and alcohol preferences in OECD countries.

Data was collected from World Health Organization databases - the Global Health Observatory data repository (GHO, 2019), specifically alcohol consumption per capita (aged 15 years and over) in litres of pure alcohol. The alcohol consumption was determined into groups of alcoholic beverages: (i) beer - barley beer 5\%; (ii) wine - grape wine $12 \%$, grape must $9 \%$, vermouth 16\%; (iii) spirits - distilled spirits 40\%, spirit-like 30\%; (iv) other - sorghum, millet, maize beers $5 \%$, cider $5 \%$, fortified wine $17 \%$ and $18 \%$, fermented wheat and fermented rice $9 \%$, other fermented beverages 9\%; (v) all - the sum of all the types of alcohol mentioned, i.e. total consumption. The analyses included 36 OECD countries (the number of member countries in 2019, when the data was collected) and the period that was analysed was from 2010 to 2018. The individual countries were divided into the relevant regions and abbreviated according to ISO 3166-1 (alpha-3): Central Europe (CE) - Austria (AUT), Czech Republic (CZE), Germany (DEU), Hungary (HUN), Poland (POL), Slovak Republic (SVK), Slovenia (SVN), Switzerland (CHE); Eastern Europe (EE) - Estonia (EST); Latvia (LVA); Lithuania (LTU); Northern Europe (NE) - Denmark (DNK), Finland (FIN), Iceland (ISL), Norway (NOR), Sweden (SWE); Southern Europe (SE) - Greece (GRC), Italy
(ITA), Portugal (POR), Spain (ESP); Western Europe (WE) Belgium (BEL), France (FRA), Ireland (IRL), Luxembourg (LUX), Netherlands (NDL), United Kingdom (GBR); North America (NA) - Canada (CAN), Mexico (MEX), United States (USA); Asia (AS) - Israel (ISR), Japan (JPN), South Korea (KOR), Turkey (TUR); South America (SA) - Chile (CHL); Oceania - Australia (AUS); New Zealand (NZL).

In the first part of the analytical processes, a descriptive analysis was used to present the variables that were analysed. This was followed by testing of differences. In the first step the Shapiro-Wilk normality test (W) was applied to support the appropriateness of selecting a non-parametric Kruskal-Wallis Rank Sum test (K-W). Subsequently, a cluster analysis was used, the aim of which was to create homogeneous groups of countries in terms of preferences for the type of alcoholic beverages for consumption.

\section{RESULT}

The preference for a certain type of alcoholic beverage is largely conditioned by the culture of each country and its geographical location, history, or social environment. Therefore, the existence of differences between countries can be expected.

Focusing on Table 1, the last row shows the tests of difference, and it is clear that the Shapiro-Wilk test recommends the use of a non-parametric method. The Kruskal-Wallis test determines the significant difference between countries, but no significant difference was confirmed between individual years. Looking at the outputs of the central tendency, the highest mean values were found in beer $(95 \%$ CI $=3.57-3.88$ ) and, conversely, the lowest values can be seen in other types of alcoholic beverages (95\% CI $=0.46-0.72)$. Of the common alcoholic beverages, the consumption of

\begin{tabular}{|c|c|c|c|c|c|}
\hline Statistic & All & Beer & Wine & Spirits & Other \\
\hline Mean & 9.21 & 3.73 & 2.77 & 2.13 & 0.59 \\
\hline $95 \% \mathrm{Cl}$ & $8.90-9.52$ & $3.57-3.88$ & $2.58-2.95$ & $1.97-2.28$ & $0.46-0.72$ \\
\hline Median & 9.52 & 3.77 & 2.86 & 1.76 & 0.12 \\
\hline Std. Dev. & 2.83 & 1.43 & 1.68 & 1.38 & 1.18 \\
\hline Minimum & 1.38 & 0.79 & 0.07 & 0.12 & 0.00 \\
\hline Maximum & 17.77 & 6.93 & 7.41 & 7.47 & 6.56 \\
\hline Range & 16.39 & 6.14 & 7.34 & 7.35 & 6.56 \\
\hline IQR & 3.15 & 1.95 & 2.16 & 1.38 & 0.70 \\
\hline Skewness & -0.46 & 0.07 & 0.30 & 1.34 & 3.54 \\
\hline Kurtosis & 1.24 & -0.52 & -0.16 & 1.98 & 14.09 \\
\hline W & $0.96 \dagger$ & $0.98 * *$ & $0.97 \dagger$ & $0.89+$ & $0.53+$ \\
\hline K-W Country $x^{2}$ & $308.59+$ & $313.75+$ & $313.45+$ & $318.20+$ & $319.36+$ \\
\hline K-W Year $X^{2}$ & 0.57 & 0.46 & 0.12 & 0.41 & 0.06 \\
\hline
\end{tabular}

Table 1 | Descriptive analysis and difference test - country and year (from 2010 to 2018)

Note: ** p-value $<0.01$; † p-value $<0.001$.

Source: original processing based on $\mathrm{GHO}$ (2019) data 

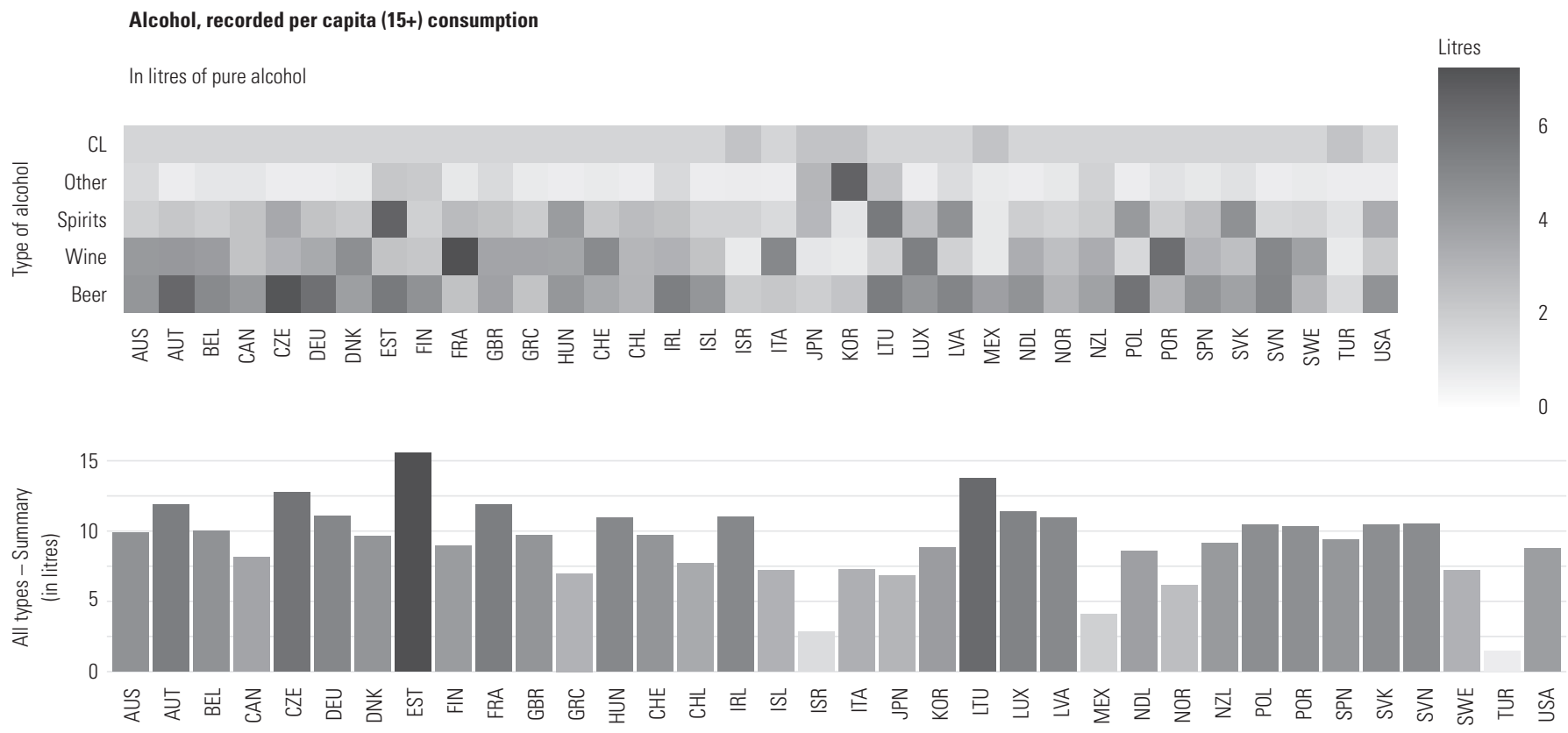

Country

Figure 1 | Average level of alcohol consumption in OECD countries (from 2010 to 2018)

Source: original processing based on $\mathrm{GHO}$ (2019) data

spirits is the lowest in terms of mean values (95\% CI $=1.97-$ 2.28). All values are in litres of pure alcohol per person aged 15 years and over. The lowest values of beer consumption (minimum $=0.79$ ) and total alcohol consumption (minimum $=1.38$ ) were found in Turkey. The lowest value of wine consumption can be seen in Israel (minimum $=0.07$ ) and the lowest value of spirits consumption was found in Mexico (minimum = 0.12). From the opposite point of view, Estonia showed the highest value of spirits consumption and total alcohol consumption (maximum = Spirits - 7.47; All - 17.77). The Czech Republic proved to be the dominant country in terms of beer consumption (maximum $=6.93$ ), while France dominated in the case of wine consumption (maximum $=7.41$ ). In the group of other alcoholic beverages, Korea was in the first place with the highest consumption (maximum $=6.56$ ). Figure 1 shows the alcohol consumption in the classification of individual beverages and the total alcohol consumption in OECD countries.

Figure 1 shows the frequency of the consumption of individual types of alcoholic beverages and the total alcohol consumption. The individual outputs can be interpreted in such a way that the darker the shade, the higher the incidence. As can be seen, Estonia, Lithuania, and the Czech Republic were the countries with the highest levels of total alcohol consumption. Simultaneously, Estonia dominated in spirits and the Czech Republic in beer. On the other hand, countries such as Turkey, Israel, and Mexico reported the lowest levels of total alcohol consumption. Figure 2 (heatmap with clusters) shows the interconnection of individual groups and subgroups of clusters, which are formed from countries and types of alcohol, and thus it shows the interconnection of groups of countries and groups of alcohol.
Prior to the cluster analysis, the silhouette method was used to estimate the most appropriate number of clusters. In terms of the consumption of individual types of alcoholic beverages, the output of this method recommends dividing OECD countries into two groups (clusters). The first cluster includes the vast majority of the countries that were analysed (SPN, CAN, USA, HUN, SVK, LVA, POL, NDL, ISL, FIN, AUT, DEU, IRL, CZE, EST, LTU, DNK, CHE, LUX, BEL, AUS, SVN, SWE, GRC, NOR, CHL, GBR, NZL, ITA, FRA, POR) and the second cluster consists of five countries, namely ISR, TUR, MEX, JPN, and KOR. On the basis of the agglomerative coefficient (0.8798), Ward's method of hierarchical clustering was the most appropriate method for identifying clusters. Regarding the consumption of other types of alcoholic beverages, the first cluster showed an average value of 0.38 , and the second cluster showed an average value of 1.84, while the dominant value was shown by Korea (mean $=6.37$ ) and Turkey had zero consumption. In the case of the consumption of wine, spirits, and beer, the first cluster (mean $=$ Wine -3.19 ; Spirits -2.31 ; Beer -4.03 ) dominates over the second cluster (mean $=$ Wine -0.16 ; Spirits -0.96 ; Beer - 1.84). On the other hand, very low wine consumption can be found in the countries of the second cluster. Also, countries such as Turkey, Mexico and South Korea were characterized by low levels of spirits consumption. The dendrogram shown in Figure 2 also shows the clusters that divide the countries into smaller groups, indicating the convergence of specific countries. It is also necessary to point out the estimate of alcoholic beverage clusters, from which it is clear that the consumption of beer and spirits showed the closest links, followed by the consumption of wine, and the group of other types of alcoholic beverages formed another but more distant category. 


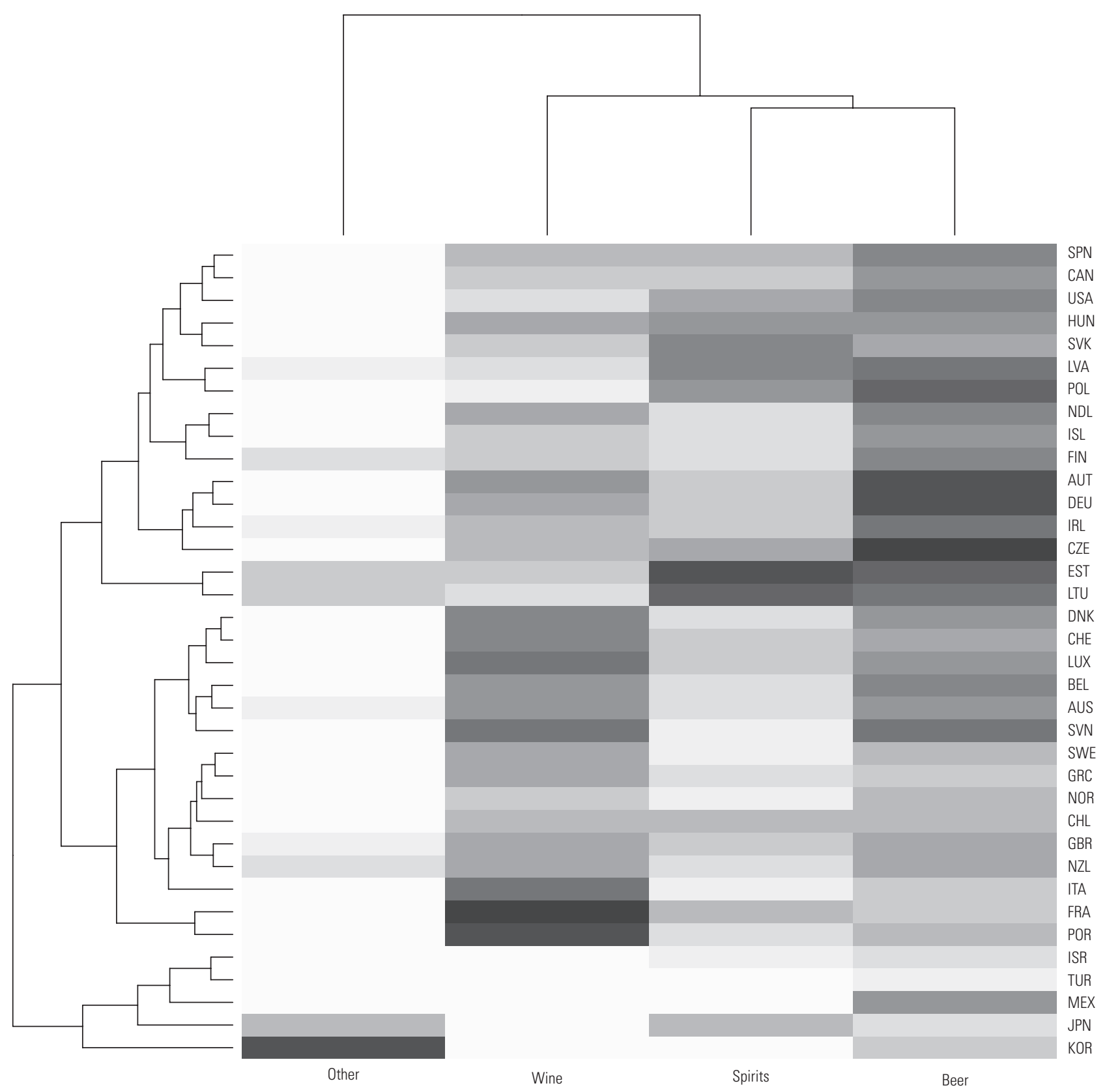

Figure 2 | Cluster analysis - types of alcoholic beverages (from 2010 to 2018) Source: original processing based on $\mathrm{GHO}(2019)$ data

On the basis of the outputs in the last row of Table 2, the significant differences between the individual regions grouping the OECD countries that were analysed can be confirmed. Eastern Europe dominated in total alcohol consumption and beer consumption, as well as spirits consumption $(95 \%$ CI = All - 12.44-14.38; Beer - 4.95-5.38; Spirits - 4.86-5.76). In the case of wine consumption, the countries of Southern Europe formed the dominant region with the highest values $(95 \% \mathrm{CI}=3.71-4.65)$. Finally, Asia showed the highest values for the consumption of other alcoholic beverages (95\% CI = 1.37-3.16).

\section{DISCUSSION AND CONCLUSION}

Alcohol is currently one of the most accessible and widespread addictive substances in the world, and at the same time it is accepted in many social communities because of its strong tradition. Mild and excessive alcohol consumption is a significant phenomenon that has accompanied humanity since ancient times and often polarizes society. Therefore, alcohol consumption is one of the main topics in many public and professional discussions focused on the health and economic effects of alcohol on society. Alcohol can be a source of economic development in countries, but these economic gains do not exceed the losses caused by alcohol consumption. The negative effects of alcohol on the health of the population are well known (Rehm et al., 2010; Smyth 


\begin{tabular}{|c|c|c|c|c|c|c|c|c|c|c|}
\hline \multirow{2}{*}{$\begin{array}{l}\text { Region / Types } \\
\text { of alcohol }\end{array}$} & \multicolumn{2}{|r|}{ All } & \multicolumn{2}{|c|}{ Beer } & \multicolumn{2}{|c|}{ Wine } & \multicolumn{2}{|c|}{ Spirits } & \multicolumn{2}{|c|}{ Other } \\
\hline & Mean & $95 \% \mathrm{Cl}$ & Mean & $95 \% \mathrm{Cl}$ & Mean & $95 \% \mathrm{Cl}$ & Mean & $95 \% \mathrm{Cl}$ & Mean & $95 \% \mathrm{Cl}$ \\
\hline AS & 4.99 & $3.97-6.01$ & 1.43 & $1.28-1.57$ & 0.16 & $0.14-0.18$ & 1.15 & $0.84-1.45$ & 2.27 & $1.37-3.16$ \\
\hline CE & 10.96 & $10.74-11.19$ & 4.99 & $4.69-5.3$ & 3.20 & $2.91-3.48$ & 2.70 & $2.43-2.97$ & 0.07 & $0.04-0.11$ \\
\hline$\overline{E E}$ & 13.41 & $12.44-14.38$ & 5.16 & $4.95-5.38$ & 1.48 & $1.3-1.66$ & 5.31 & $4.86-5.76$ & 1.46 & $1.21-1.72$ \\
\hline SA (Chile) & 7.71 & $7.41-8.02$ & 2.67 & $2.54-2.8$ & 2.66 & $2.5-2.82$ & 2.38 & $2.13-2.64$ & 0.00 & 0.00 \\
\hline$\overline{N A}$ & 7.00 & $6.16-7.85$ & 3.84 & $3.73-3.96$ & 1.27 & $0.95-1.58$ & 1.77 & $1.29-2.24$ & 0.13 & $0.09-0.18$ \\
\hline $\mathrm{NE}$ & 7.83 & $7.43-8.22$ & 3.43 & $3.22-3.64$ & 2.78 & $2.48-3.08$ & 1.23 & $1.16-1.29$ & 0.39 & $0.2-0.58$ \\
\hline$\overline{O C}$ & 9.52 & $9.28-9.77$ & 3.72 & $3.53-3.9$ & 3.40 & $3.21-3.6$ & 1.39 & $1.32-1.45$ & 1.02 & $0.93-1.10$ \\
\hline SE & 8.49 & $7.95-9.03$ & 2.65 & $2.33-2.97$ & 4.18 & $3.71-4.65$ & 1.50 & $1.31-1.7$ & 0.16 & $0.11-0.20$ \\
\hline$\overline{W E}$ & 10.44 & $10.12-10.76$ & 3.94 & $3.68-4.21$ & 4.17 & $3.76-4.58$ & 1.98 & $1.86-2.1$ & 0.35 & $0.25-0.44$ \\
\hline K-W Region $\mathrm{X}^{2}$ & \multicolumn{2}{|r|}{$223.89+$} & \multicolumn{2}{|c|}{$190.95 \dagger$} & \multicolumn{2}{|c|}{$200.31 \dagger$} & \multicolumn{2}{|c|}{$153.32 \dagger$} & \multicolumn{2}{|c|}{$128.65 \dagger$} \\
\hline
\end{tabular}

Table 2 | Descriptive analysis and difference test - regions (from 2010 to 2018) Note: $†$ p-value $<0.001$.

Source: original processing based on $\mathrm{GHO}$ (2019) data

et al., 2015), as confirmed by the findings of many international studies that have examined the health risks of alcohol consumption (Klein et al., 2020; McClure et al., 2013; Roerecke et al., 2019; Scherubl, 2019; Scoccianti et al., 2013). The consumption of different types of alcoholic beverages poses different risks (Kerr et al., 2000), and this negative effect on health is often reflected in increased alcohol-related mortality, which contributes to the global mortality burden (Rehm \& Shield, 2013). Last but not least, there is a need to discuss the economic losses caused by alcohol consumption, which lead to increased health and social costs, lost productivity, and declining GDP (Jyani et al., 2019; Laramee et al., 2013; Ranaweera et al., 2018; Verhaeghe et al., 2017). On the basis of these facts, there is a need to address the issue of alcohol in different countries and to identify the current situation. Therefore, the primary objective of this study was to determine the differences in alcohol consumption and alcohol preferences in OECD countries. This objective was fulfilled through several analytical processes, in which descriptive analysis, difference analysis, and cluster analysis were applied.

On the evidence of the outputs of the above-mentioned analyses, it can be stated that Estonia, Lithuania, and the Czech Republic are among the countries with the highest alcohol consumption. Simultaneously, Lyszczarz (2019) revealed the highest costs of alcohol-related mortality for countries such as Lithuania and Estonia, reflecting the fact that these countries have great potential for improving their alcohol policy and alcohol control. On the other hand, countries such as Turkey, Israel, and Mexico are characterized by low total alcohol consumption during the period that was analysed. These findings are in line with the findings of a study conducted by Parna (2019), and similar results were reported by the OECD (2019). Very similar outputs were also already presented by Popova et al. (2007), in which the authors point out very strongly the harmful effect of excessive alcohol consumption.

The outputs of this study also show that there was no significant change in the reduction of alcohol consumption over time in the countries that were analysed. In any case, the differences between OECD countries in terms of total alcohol consumption and preferred alcoholic beverages are undeniable. These differences can be explained by culture or religion, as evidenced by the low values in Turkey or Israel and the high values in the Czech Republic. Another explanation for the differences revealed is the lifestyle, as evidenced by the high consumption of alcohol in Estonia, but in that country, it is also possible to discuss its culture. Regarding the regions that were analysed, Eastern Europe (Estonia, Latvia, Lithuania) showed the highest rates of total alcohol consumption, as well as beer and spirits consumption. Asian countries (Israel, Japan, Korea, Turkey) showed the lowest rates of consumption of common alcoholic beverages (beer, wine, spirits) and the highest rates of consumption of other alcoholic beverages. In Southern Europe (Greece, Italy, Portugal, Spain), wine consumption dominated, which agrees with the division of countries according to the preferences for the type of alcoholic beverages in the study conducted by Bentzen and Smith (2018).

The output of the cluster analysis in this study divided OECD countries into two basic groups in terms of the consumption of beer, wine, spirits, and other alcoholic beverages. The first group consisted of all the countries except Israel, Turkey, Mexico, Japan, and South Korea; these countries formed the second group. Compared to the second group, the first group dominated in the consumption of wine, spirits, and beer. With a focus on the individual countries of the first group, beer consumption was preferred in the Czech Republic, wine consumption was dominant in France, and spirits consumption was particularly characteristic of Estonia. Within the second group, the highest preference for other types of alcoholic beverages occurred in South Korea (sorghum, millet, maize beers 5\%, cider $5 \%$, fortified wine $17 \%$ and $18 \%$, fermented wheat and fermented rice $9 \%$, other fermented beverages $9 \%$ ), reflecting the specific culture of this country. The countries of the second group showed a very low preference for wine, and countries such as Korea, Mexico, and Turkey preferred spirits at a lower rate. 
These findings help in the effective development of an intervention strategy for alcohol control in developed countries. In order to improve the health of the population and contribute to economic development, policymakers need to focus on the issue of alcohol consumption. Public policy instruments such as high prices, taxes, or a ban on alcohol promotion can be effective in reducing total alcohol consumption (Anderson et al., 2009), which can lead to lower alcohol-related mortality and economic gains. However, legislative measures alone are not enough. The cooperation of the whole society can be very effective in this issue. Healthy lifestyle education and prevention play an important role in addressing this real threat. Drinking spirits and beer is the riskiest, and therefore education and prevention should focus on areas with a higher consumption of these types of alcoholic beverages. Last but not least, an efficient health system and healthcare financing can help eliminate the consequences of excessive alcohol consumption (Stefko et al., 2017).

The limitations of this study lie in the fact that the data also includes the consumption of alcohol by people who are not citizens of the countries in question (tourism, migration), and therefore the results could be affected, but this effect cannot be expected to be significant. Simultaneously, the division of countries into regions did not include all countries, especially outside Europe. On this basis, the results of non-European regions need to be carefully considered. Further research efforts will focus on examining the relations between alcohol consumption in the classification of individual beverages and health outcomes, as well as economic indicators.
Authors' contributions: BG prepared the econometric design of the study, $\mathrm{VI}$ performed the literature review and the data mining procedure. The data analysis was performed by MR. BG, MR, and VI participated in the data interpretation. All the authors contributed to the draft and approved the final manuscript before its submission.
Declaration of interest: No conflict of interest

\section{REFERENCES}

Anderson, P., Chisholm, D., \& Fuhr, D. C. (2009). Alcohol and Global Health 2 Effectiveness and cost-effectiveness of policies and programmes to reduce the harm caused by alcohol. Lancet, 373(9682), 2234-2246. http://doi.org/10.1016/S0140-6736(09)60744-3

Bagnardi, V., Rota, M., Botteri, E., Tramacere, I., Islami, F., Fedirko, V., Scotti, L., Jenab, M., Turati, F., Pasquali, E., Pelucchi, C., Bellocco, R., Negri, E., Corrao, G., Rehm, J., Boffetta, P., \& La Vecchia, C. (2013). Light alcohol drinking and cancer: a meta-analysis. Annals of Oncology, 24(2), 301-308. http://doi.org/10.1093/annonc/mds337

Bencevic-Striehl, H., Malatestinic, D., \& Vuletic, S. (2009). Regional Differences in Alcohol Consumption in Croatia. Collegium Antropologicum, 33, 39-41.

Bentzen, J., \& Smith, V. (2018). Structural Changes in the Consumption of Beer, Wine and Spirits in OECD Countries from 1961 to 2014. Beverages, 4(1), 8. http://doi.org/10.3390/beverages4010008

Branstrom, R., \& Andreasson, S. (2008). Regional differences in alcohol consumption, alcohol addiction and drug use among Swedish adults. Scandinavian Journal of Public Health, 36(5), 493-503. http://doi.org/10.1177/1403494807087557

Callinan, S., Livingston, M., Room, R., \& Dietze, P. (2016). Drinking contexts and alcohol consumption: How much alcohol is consumed in different Australian locations? Journal of Studies on Alcohol and Drugs, 77(4), 612-619. http://doi.org/10.15288/jsad.2016.77.612

Devaux, M., \& Sassi, F. (2015). Alcohol consumption and harmful drinking: Trends and social disparities across OECD countries. OECD Health Working Papers, No. 79. Paris: OECD Publishing. http://doi.org/10.1787/5js1qwkz2p9s-en.

Dey, M., Gmel, G., Studer, J., Dermota, P., \& Mohler-Kuo, M. (2014). Beverage preferences and associated drinking patterns, consequences and other substance use behaviours. European Journal of Public Health, 24(3), 496-501. http://doi.org/10.1093/eurpub/ckt109

GHO. (2019). World Health Organization databases - Global Health Observatory data repository. Available at: https://www.who.int/gho/database/en/
Jyani, G., Prinja, S., Ambekar, A., Bahuguna, P., \& Kumar, R. (2019). Health impact and economic burden of alcohol consumption in India. International Journal of Drug Policy, 69, 34-42. http://doi.org/10.1016/j.drugpo.2019.04.005

Kerr, W. C., Fillmore, K. M., \& Marvy, P. (2000). Beverage-specific alcohol consumption and cirrhosis mortality in a group of English-speaking beer-drinking countries. Addiction, 95(3), 339-346. http://doi.org/10.1046/j.1360-0443.2000.9533394.x

Klein, W. M. P., Jacobsen, P. B., \& Helzlsouer, K. J. (2020). Alcohol and cancer risk clinical and research implications. JAMA: Journal of the American Medical Association, 323(1), 23-24. http://doi.org/10.1001/jama.2019.19133

Kossova, T., Kossova, E., \& Sheluntcova, M. (2017). Investigating the volume and structure of alcohol consumption in Russian regions. Journal of Economic Studies, 44(2), 266-281. http://doi.org/10.1108/JES-07-2015-0118

Laramee, P., Kusel, J., Leonard, S., Aubin, H. J., Francois, C., \& Daeppen, J. B. (2013). The economic burden of alcohol dependence in Europe. Alcohol and Alcoholism, 48(3), 259-269. http://doi.org/10.1093/alcalc/agt004

Lyszczarz, B. (2019). Production Losses Associated with Alcohol-Attributable Mortality in the European Union. International Journal of Environmental Research and Public Health, 16(19), 3536. http://doi.org/10.3390/ijerph16193536

McClure, L. A., Fernandez, C. A., Clarke, T. C., LeBlanc, W. G., Arheart, K. L., Fleming, L. E., \& Lee, D. J. (2013). Risky drinking in the older population: A comparison of Florida to the rest of the US. Addictive Behaviors, 38(4), 1894-1897. http://doi.org/10.1016/j.addbeh.2012.12.020

Mehta, N., \& Myrskyla, M. (2017). The population health benefits of a healthy lifestyle: Life expectancy increased and onset of disability delayed. Health Affairs, 36(8), 1495-1502. http://doi.org/10.1377/hlthaff.2016.1569

OECD. (2019). Alcohol consumption among adults. Health at a Glance 2019: OECD indicators. Paris: OECD Publishing. http://doi.org/10.1787/961753cf-en

Ogwang, T., \& Cho, D. I. (2009). Economic determinants of the consumption of alcoholic beverages in Canada: a panel data analysis. Empirical Economics, 37(3), 599-613. http://doi.org/10.1007/s00181-008-0248-4 
Parna, K. (2019). Alcohol consumption and alcohol policy in Estonia 2000-2017 in the context of Baltic and Nordic countries. Drug and Alcohol Review, 2019. http://doi.org/10.1111/dar.13008

Pelucchi, C., Tramacere, I., Boffetta, P., Negri, E., \& La Vecchia, C. (2011). Alcohol Consumption and Cancer Risk. Nutrition and Cancer-An International Journal, 63(7), 983-990. http://doi.org/10.1080/01635581.2011.596642

Popova, S., Rehm, J., Patra, J., \& Zatonski, W. (2007) Comparing alcohol consumption in central and eastern Europe to other European countries. Alcohol and Alcoholism, 42(5), 465-473. http://doi.org/10.1093/alcalc/agl124

Probst, C., Kilian, C., Sanchez, S., Lange, S., \& Rehm, J. (2020). The role of alcohol use and drinking patterns in socioeconomic inequalities in mortality: a systematic review. Lancet Public Health, 5(6), e324-e332. http://doi.org/10.1016/S2468-2667(20)30052-9.

Ranaweera, S., Amarasinghe, H., Chandraratne, N., Thavorncharoensap, M., Ranasinghe, T., Karunaratna, S., Kumara, D., Santatiwongchai, B., Chaikledkaew, U., Abeykoon, P., \& De Silva, A. (2018). Economic costs of alcohol use in Sri Lanka. Plos One, 13(6), e0198640. http://doi.org/10.1371/journal.pone.0198640

Rehm, J., Baliunas, D., Borges, G. L. G., Graham, K., Irving, H., Kehoe, T., Parry, C. D., Patra, J., Popova, S., Poznyak, V., Roerecke, M., Room, R., Samokhvalov, A. V., \& Taylor, B. (2010). The relation between different dimensions of alcohol consumption and burden of disease: an overview. Addiction, 105(5), 817-843. http://doi.org/10.1111/j.1360-0443.2010.02899.x

Rehm, J., \& Shield, K. D. (2013). Alcohol and mortality global alcoholattributable deaths from cancer, liver cirrhosis, and injury in 2010. Alcohol Research-Current Reviews, 35(2), 174-183

Roerecke, M., Vafaei, A., Hasan, O. S. M., Chrystoja, B. R., Cruz, M., Lee, R., Neuman, M. G., \& Rehm, J. (2019). Alcohol consumption and risk of liver cirrhosis: A systematic review and meta-analysis. American Journal of Gastroenterology, 114(10), 1574-1586. http://doi.org/10.14309/ajg.0000000000000340

Scherubl, H. (2019). Alcohol use and cancer risk. Deutsche Medizinische Wochenschrift, 144(19), 1354-1360. http://doi.org/10.1055/a-0928-0586

Scoccianti, C., Straif, K., \& Romieu, I. (2013). Recent evidence on alcohol and cancer epidemiology. Future Oncology, 9(9), 1315-1322. http://doi.org/10.2217/fon.13.94

Smyth, A., Teo, K. K., Rangarajan, S., O’Donnell, M., Zhang, X., Rana, P., Leong, D. P., Dagenais, G., Seron, P., Rosengren, A., Schutte, A. E., Lopez-Jaramillo, P., Oguz, A., Chifamba, J., Diaz, R., Lear, S., Avezum, A., Kumar, R., Mohan, V., Szuba, A., Wei, L., Yang, W., Jian, B., McKee, M., \& Yusuf, S. (2015). Alcohol consumption and cardiovascular disease, cancer, injury, admission to hospital, and mortality: a prospective cohort study. Lancet, 386(10007), 1945-1954. http://doi.org/10.1016/S0140-6736(15)00235-4

Spach, M. (2016). Economic issues and public alcohol abuse prevention policies in France. Santé Publique, 28(4), 461-470. http://doi.org/10.3917/spub.164.0461

Stefko, R., Jencova, S., Litavcova, E., \& Vasanicova, P. (2017). Management and funding of the healthcare system. Polish Journal of Management Studies, 16(2), 266-277. http://doi.org/10.17512/pjms.2017.16.2.23

Thavorncharoensap, M., Teerawattananon, Y., Yothasamut, J., Lertpitakpong, C., Thitiboonsuwan, K., Neramitpitagkul, P., \& Chaikledkaew, U. (2010). The economic costs of alcohol consumption in Thailand, 2006. BMC Public Health, 10, 323. http://doi.org/10.1186/1471-2458-10-323

Thun, M. J., Peto, R., Lopez, A. D., Monaco, J. H., Henley, S. J., Heath, C. W., \& Doll, R. (1997). Alcohol consumption and mortality among middle-aged and elderly US adults. New England Journal of Medicine, 337(24), 1705-1714. http://doi.org/10.1056/NEJM199712113372401

Verhaeghe, N., Lievens, D., Annemans, L., Vander Laenen, F., \& Putman, K. (2017). The health-related social costs of alcohol in Belgium. BMC Public Health, 17, 958. http://doi.org/10.1186/s12889-017-4974-4 